\title{
AsEsoramiEnTO SEXUAL EN PERSONA SORDOCIEGA \\ A PARTIR DEL CONTROL DE CONTINGENCIAS Y \\ TÉCNICAS MENTALIZADORAS
}

\section{Deafblind Sexual Counseling by Means of Contingency Control and Mentalizing Techniques: A Case Study}

\author{
Luisa Fernanda Suárez Monsalve ${ }^{1}$ \\ https://doi.org/10.17533/udea.rp.v12n1a07
}

\section{Resumen}

Este estudio de caso describe un asesoramiento sexual con paciente sordociego, quien presentaba conductas sexuales no identificadas por él mismo como disfuncionales, debido a su baja capacidad de juicio y empatía. Para el tratamiento se emplearon terapias con evidencia empírica y se entrenó a los padres del paciente en un programa de control de contingencias. Para la evaluación se empleó las Historias extrañas de Happé. Los resultados fueron: extinción de las conductas de tocamiento, la formación de representaciones mentales asociadas a temas de educación sexual y desarrollo de algunas habilidades mentalizadoras. Se concluye que la persona con

Recibido: 2020-06-13/Aceptado: 2020-08-28

Para citar este artículo en APA: Suárez Monsalve, L. (2020). Asesoramiento sexual en persona sordociega a partir del control de contingencias y técnicas mentalizadoras. Revista de Psicología Universidad de Antioquia, 12(1), 135-155. doi: https:// doi.org/10.17533/udea.rp.v12n1a07 sordoceguera participante del estudio de caso presentaba dificultades para el desarrollo de habilidades metacognitivas, no solo por la ausencia de información visual y auditiva que le ha impedido formar representaciones autónomas de su entorno, a su vez por su nula educación sexual; aspectos que se pueden solucionar con terapia mentalizadora y un programa de educación sexual, con los ajustes necesarios para permitir el acceso cognitivo y sensorial a este programa.

Palabras clave: sordoceguera, discapacidad sensorial, mentalización, psicoterapia, sexualidad. 


\begin{abstract}
This case study describes sexual counseling with a deafblind patient who had shown sexual behaviors not identified by him as dysfunctional, due to his low capacity for judgment and empathy. For treatment, empirical evidence therapies were implemented, and his parents were trained in a contingency control program. For the evaluation, Happé's Stranges Stories test were used. The results were: extinction of touching behaviors, construction of mental representations associated with sexual education issues, and development of some mentalizing skills. Conclusion: the
\end{abstract}

patient presented difficulties for the development of meta-cognitive skills, not only because of the visual and auditory information absence, preventing the formation of autonomous representations of its surroundings, but also for his null sex education. These aspects can be solved with mentalizing therapy and an adapted sexual education program, which allows to the patient cognitive and sensory access to the program.

Keywords: deafblindness, sensory disabilities, mentalization, psychotherapy, sexuality.

\title{
Introducción
}

La sordoceguera es una falta de capacidad auditiva y visual, la cual puede ser total o parcial en ambos órganos; esta limitación sensorial, según la ley 982 de 2005 — por la cual se establecen normas tendientes a la equiparación de oportunidades para las personas sordas y sordociegas y otras disposiciones-, genera falencias comunicacionales, orales, de movilidad y dificultad para acceder a la información.

Si bien los problemas relacionados con los aspectos sexuales han presentado una cantidad importante de hallazgos científicos, se encuentra que la mayoría de estos se interesan por el estudio de poblaciones con enfermedades mentales o poblaciones no clínicas, siendo este interés limitado en lo referente al aprendizaje, desarrollo sexual y las conductas sexuales funcionales o disfuncionales que pueden presentarse en personas en situación de discapacidad. En la población sordociega, aunque se encuentran investigaciones — principalmente sobre el modo en que funciona la teoría de la mente en personas sordas (Quintana, 2004) — que aportan a la sugerencia de planes de educación sexual (López, 2002), es poca la evidencia disponible donde se expliquen los procesos metacognitivos de las personas sordociegas, para fortalecer estos planes.

En el apartado Otros encuentros de los servicios sanitarios para asesoramiento y consejo médico de la quinta edición del Manual diagnóstico y estadístico 
de los trastornos mentales (DSM 5, 2014) se establece el Asesoramiento sexual (V65.49) como un servicio utilizado cuando el individuo desea orientación en educación y comportamiento sexual, actitudes sexuales, juegos sexuales o cualquier otro asunto relacionado con el sexo, directamente con el paciente o en consideración de un tercero. Por su parte, en la décima edición de la Clasificación Internacional de Enfermedades (CIE 10, 2016) este modo de asesoramiento se cataloga como Consulta sexual no especificada (Z70.9). Esta categoría se incluye para codificar los trastornos sexuales que no cumplen los criterios para un trastorno sexual específico y que no constituyen una disfunción sexual ni una parafilia.

Anderson y Cole (2016), en su investigación sobre asesoramiento sexual con personas con discapacidad física, encontraron que en esta población los aspectos sexuales han sido descuidados, puesto que son míticamente identificados como personas no sexuales, que no tienen necesidad de expresión sexual, generando así, incluso, desconocimiento en los profesionales en el asesoramiento dirigido a esta población.

Estos autores explican que la vida social y sexual de las personas con discapacidad requiere de un abordaje, por parte de los profesionales, en aspectos de educación social-sexual; para ello refieren intervenciones con el fin de aumentar el nivel de desarrollo y reconocimiento de los sentimientos y actitudes hacia los temas sociales-sexuales. Se propone que la construcción de estos programas debe contemplar la comprensión de las condiciones de igualdad, en derechos y responsabilidades, con las personas sin discapacidad.

Por su parte, Lehane, Dammeyer, Hovaldt y Elsass (2017), en su estudio sobre sexualidad y bienestar en parejas que viven con sordoceguera adquirida, hallaron dificultades en esta área, representadas por un bajo nivel de actividad sexual y pobre bienestar psicológico derivado de esta condición.

Diferente a un niño sin discapacidad visual o auditiva que percibe y responde a la estimulación tanto social como verbal, el infante sordociego inicia su experiencia vital en un estado de privación sensorial que conduce al niño a un aislamiento social, pues la pérdida de la lengua oral y de la captación visual total del ambiente crea limitaciones para la exploración directa del mundo, y sobre todo para el acceso al aprendizaje social (Anderson y Cole, 2016). 
Posteriormente, el adolescente con sordoceguera debe trabajar fuertemente para acoplar su comportamiento social dentro de un contexto específico. Algunos comportamientos de las personas con esta discapacidad implican contacto físico permanente, por la necesidad de contar con información táctil. Ahora, la regulación comportamental que se les llega a exigir a las personas con sordoceguera podrá generar como resultado que estas cuenten con poco acceso a la información táctil, disminuyendo su habilidad para desempeñarse en sus interacciones (Anderson y Cole, 2016).

Wellan y Peterson (2013) encontraron dificultades interaccionales en personas con sordera, con cuidadores oyentes no señantes, al momento de resolver ejercicios de teoría de la mente (ToM), la cual es definida por estos autores como la «capacidad de comprender, explicar y predecir el comportamiento de otras personas a través del proceso de realizar inferencias acerca de estados mentales, intenciones, deseos y creencias» (p. 324).

Peterson refiere que las diferencia

\begin{abstract}
A la hora de realizar interacciones con el entorno social que facilitan el acceso a las mentes de los demás y a la suya propia en estas poblaciones [sordas] son bien distintas, se plantea la posibilidad de si estas poblaciones son capaces de establecer rutas alternativas para acceder a habilidades representacionales que les permitan aprender sobre cierta clase de pensamientos sin recurrir al lenguaje proposicional, lo que ayudaría a adquirir, aunque sea más tardíamente una ToM (citado en Quintana, 2004, p.115).
\end{abstract}

En la misma línea, Wellan y Peterson (2013) concluyen que la comunicación tanto no verbal como verbal estimulan múltiples componentes de la ToM, siendo así la vivencia comunicativa pieza clave en su progreso.

Según Peterson y Siegal, en los nińos sordos con cuidadores oyentes no señantes se puede generar una deficiencia en la comprensión de algunos estados de la mente, todo ello por causa de la falta de interacción conversacional:

Las madres oyentes, aunque conozcan la lengua de signos, tienen un dominio de ésta no equiparable al de un hablante nativo, esto hace que en sus intercambios conversacionales limiten la comunicación con su hijo sordo a percepciones simples y a algunas emociones básicas, lo que conduce a que los nińos sordos se formen una noción del mundo similar a la que se percibe a través de una cámara 
de video y, por tanto, se dificulta su acceso a aquella información que versa sobre pensamientos y sentimientos ajenos y a un vocabulario de términos mentalistas necesarios para el desarrollo de la teoría de la mente (citado en Quintana, 2004, p. 112).

Descritas estas características sobre la sexualidad y las dinámicas relacionales de personas con discapacidad visual o auditiva, por lo que se refiere al momento de la intervención asociada al área sexual, ya sea en una persona con o sin condición de discapacidad, entre las estrategias más adecuadas está acudir a las guías clínicas que contienen las intervenciones basadas en la evidencia.

La práctica psicológica basada en la evidencia (PPBE) tiene el fin de maximizar la eficacia y los resultados favorables en las intervenciones psicológicas, conduciendo a la generalización de prácticas que permitan impactar en áreas de intervención en muchas poblaciones. Las PPBE plantean la integración de tres variables al momento de realizar una intervención psicológica: (a) la utilización de la mejor evidencia disponible que provea la psicología para la toma de decisiones de intervención durante el desarrollo de tratamientos psicológicos; (b) considerar las preferencias y características culturales de la población a la cual se dirige la intervención; e (c), integrar la experiencia profesional del psicólogo (Martínez-Taboas, 2014).

Desde las PPBE se plantea que para la intervención de la conducta sexual disfuncional se puede acudir a la combinación de tres intervenciones psicológicas diferentes. La primera estrategia es educar al paciente. Colom (2011) presentó una serie de evidencias acerca de la efectividad de la implementación de la psicoeducación en trastornos psicológicos, indicando que es imprescindible contar con programas de educación terapéutica con todo tipo de pacientes, incluyendo a aquellos con patologías no psicológicas como cardiopatías, enfermedades respiratorias, diabetes, entre otras. En estos casos la psicoeducación demostró impactar claramente de forma positiva en las diferentes intervenciones.

En la contemporaneidad la psicoeducación forma parte de las rutinas psicoterapéuticas y muestra eficacia en la profilaxis de todo tipo de recaídas, demostrando efectos aun a los dos y a los cinco años de llevada a cabo la intervención, lo cual ha contribuido en la reducción de la duración de los episodios 
de recaída, en las hospitalizaciones y en los problemas de adherencia al tratamiento. El autor presenta la efectividad del programa de control de contingencias para trastornos del comportamiento, estableciendo el castigo, aplicado de manera contingente a la conducta problema, como variable determinante para la eficacia de este programa. Con el castigo se busca que la modificación de la conducta lleve a la persona a una respuesta funcional socialmente; esto se logra con la estimulación aversiva que independiente de la respuesta puede producir una supresión de la conducta instrumental.

Como tercera estrategia, Kassa, Luck, Bekele y Riedel-Heller (2016) presentan un listado de tratamientos basados en la evidencia y proponen que la terapia basada en la mentalización sirve para aumentar la conciencia acerca de los estados mentales de los demás y de sí mismo, tanto de forma implícita como explícita. En esta terapia se plantea aumentar la capacidad de entendimiento de los estados mentales y emocionales para crear modificaciones e influencias en comportamientos, creencias, deseos, sentimientos y pensamientos individuales y sociales, a partir del entendimiento del otro, permitiendo así la creación de bases empáticas de interacción en las relaciones sociales y personales.

Bateman y Fonagy (2016) proponen una evaluación de la forma en la que el paciente interactúa con su cuerpo, mente, familiares, comunidad, o comunidades, y entorno, debido a que son las experiencias mediante las cuales se identifica en el paciente las cuatro dimensiones de la mentalización: características internas de sí mismo; conocimiento de las características externas de sí mismo; conocimiento de las características internas de los demás; y, características externas de los demás. Las dos primeras áreas tienen un enfoque afectivo y cognitivo; las dos últimas, además de este enfoque, precisan de la habilidad de respuesta ante las reacciones de los demás, junto con la capacidad de reflexionar sobre sí mismo y los otros.

Teniendo en cuenta lo anterior, el objetivo de este reporte es presentar un análisis de caso desarrollado con terapias basadas en la evidencia, donde se pretendió intervenir las conductas sexuales disfuncionales de un paciente sordociego, empleando inicialmente técnicas de psicoeducación y la extinción de la conducta sexual disfuncional, y posteriormente la terapia basada en la mentalización. 


\section{Metodología}

Estudio analítico de caso clínico con diseño intraserie de reversión A-B-A, donde la primera $A$ es la línea base estable, la $B$ es la aplicación del tratamiento y la segunda $A$, la fase de retirada del tratamiento, esperándose que en la última fase (A) existan cambios en relación con la primera (A) por causa de B. Se utiliza una metodología de observación directa del fenómeno con muestreo por conveniencia.

\section{Datos de identificación y problema actual}

El paciente es un adulto sordociego, desempleado, soltero. Vive con sus padres y su hermano menor, de 4 años. Uno de los padres del paciente acude a consulta con él, pues presenta conductas de tocamiento con su hermano menor.

$\mathrm{Al}$ momento de la primera consulta el paciente presentaba los siguientes síntomas: conductualmente, conductas de tocamiento recurrentes; afectivamente, falta de empatía y aplanamiento emocional, pero con la capacidad interoceptiva de reconocer algunas emociones primarias; somáticamente, síntomas de excitabilidad como respuesta a la conducta de tocamiento; interpersonalmente, poca interacción con sus familiares, debido a que solamente uno de ellos manejaba la lengua de señas colombianas (LSC); y, cognitivamente, falta de representaciones mentales asociados al tema sexual, sin capacidad de juicio para anticipar lo inapropiado de sus comportamientos.

En su primer consulta, el paciente presentaba una actitud colaboradora; se encontraba orientado en las tres esferas (tiempo, espacio y persona); contaba con una apariencia prolija y ordenada; su estado de alerta y actividad motora eran adecuados; euproséxico; no cuenta con acceso sensorial a estimulaciones visuales y auditivas; presentaba baja expresión emocional y baja capacidad de juicio y raciocinio; con representaciones mentales incompletas, introspección y prospección debilitados y con una capacidad comunicacional de expresión, comprensión y producción de la LSC amplia y suficiente. El paciente no refiere historia psiquiátrica anterior, pero presenta historia médica de sordoceguera, debido a rubéola congénita y soplo en el corazón. 


\section{Historia del problema}

Durante su vida académica y familiar el paciente no tuvo acceso a programas de educación sexual; aunado a ello, experimentó diversos eventos potencialmente activadores de su conducta sexual disfuncional: a sus 6 ańos el paciente fue tocado por una joven mayor que él (12 años), posteriormente, a sus 18 ańos, ya es el paciente quien toca a una joven de doce ańos. En enero de 2016 su madre reanuda su vida laboral, por lo que debe dejarlo solo en casa con su hermano de tres años de edad; es ahí cuando el paciente comienza a practicar tocamientos con su hermano de manera recurrente al momento del juego. Aunque su madre acude a las autoridades legales, este acto no es calificado como un delito, al determinar que la persona sordociega no actuó con dolo; además, no existía verdad jurídica, por lo que no se inició un proceso penal en su contra.

En su historia personal y familiar se identifica que el paciente nace sordo en una familia de oyentes; solo uno de sus familiares comienza a aprender LS, por lo que crece alejado de las conversaciones familiares. A la edad de 5 años queda ciego y empieza a aprender lengua de señas táctil, teniendo percepción únicamente de las manos del emisor, mas no de la información táctil sobre el gesto facial que acompaña la seña. Ingresa a un colegio para personas con discapacidad, donde interactúa con otros niños sordos. En su casa se comunica, por medio de la lengua de señas táctil, únicamente con uno de los integrantes de su familia, quien por estar en diversas ocupaciones del hogar no lograba comunicarse con él de manera constante, impidiéndole informar totalmente los acontecimientos familiares cotidianos.

En su tiempo libre, el paciente comienza a compartir con algunos animales domésticos que le son regalados a la edad de 7 años y con quienes pasaba la mayor parte de su tiempo. En su adolescencia continúa en la misma institución educativa, donde establece relaciones sociales con algunas personas sordas de su edad, quienes se comunicaban por medio de lengua de señas táctil. Durante sus clases no siempre estaba disponible de manera ininterrumpida un guía intérprete exclusivamente para él como estudiante sordociego, ni se impartió un programa de educación socio-sexual en su colegio. En su adolescen- 
cia aprende a apoyar a su familia en las labores del hogar, cocinando, lavando la ropa, trapeando, etc. Al finalizar sus estudios de bachillerato, las personas sordas con quienes compartía en el colegio continúan su vida académica en estudios tecnológicos y la única persona en la familia que sabía señas encuentra trabajo, por lo que disminuye su tiempo para ser su intérprete en el entorno familiar. En su edad adulta, la familia adopta un niño de 3 años, a quien el paciente logra enseñarle algunos signos de LSC y comienza a compartir con él gran parte de su tiempo, en juegos donde se dan las prácticas de tocamiento.

\section{Instrumentos de evaluación}

Para realizar el proceso de evaluación se aplicó pre y post-test mediante un instrumento de tareas de ToM, buscando con esto medir la capacidad mentalizadora del paciente; además, se empleó una entrevista clínica y un registro de control de contingencias para establecer la frecuencia de la conducta de tocamiento dirigida a su hermano adoptivo.

- Entrevista clínica: es un instrumento cualitativo que, adaptado a los aspectos culturales, demuestra ser eficaz para comunicarse con el individuo e identificar los síntomas diagnósticos transversales y las escalas de gravedad específicas de cada diagnóstico clínico. Esta técnica está diseñada para ser utilizada en la primera evaluación, buscando establecer así el diagnóstico inicial, y en sesiones posteriores para realizar un seguimiento, fundamentando con esto la planificación del tratamiento y la modificación de síntomas a través del proceso terapéutico (Perpiñá, 2012).

- Registros de conducta: es una técnica que permite observar la frecuencia de la conducta problema, ayudando a identificar en qué situaciones ocurre y en qué intensidad. Al aplicarse en pacientes con discapacidad, esta debe hacerse con el apoyo de evaluadores entrenados o personas del entorno. El objetivo de los registros (tabla 1) es la identificación del cambio de la línea base y su relación con los refuerzos y castigos empleados (Domjan, 2010). El registro de control de contingencias (gráfico 1) es hecho por uno de los padres del paciente durante dos semanas y tres días. Se indicó que una 
vez iniciado el juego se identificara que el paciente se predispone a iniciar la conducta de tocamiento, inmediatamente el hermano fuera retirado mientras se hacia la seña de mal y se realizaba el registro del minuto en que se suspende el juego.

Gráfico 1. Registro de control de contingencias

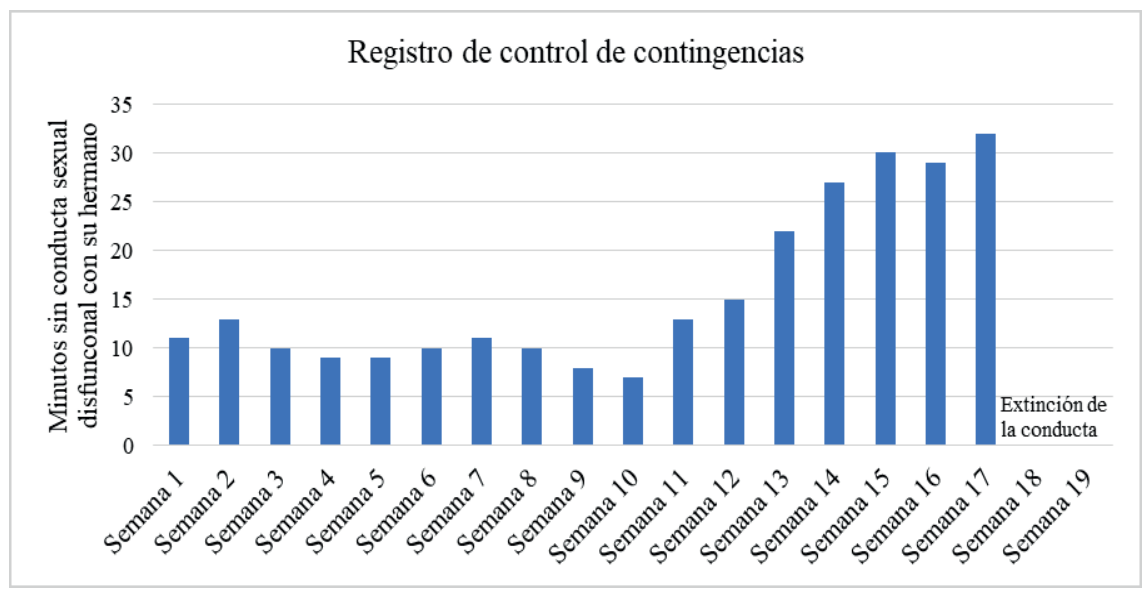

- Historias extrañas de Happé. Previo a la aplicación del método basado en la mentalización, se aplicó este instrumento, el cual es útil para valorar el rendimiento en la teoría de la mente. Para la población oyente en cada ítem se mide: la respuesta dada, tiempo de realización de la tarea, palabras con dificultad y frases con dificultad; además, se tiene un espacio de observaciones. El resultado de esta prueba permite identificar el nivel empático del paciente (Gil, Fernández, Bengoechea y Arritea, 2012; Lahera, 2007).

Dado que la prueba está estandarizada para personas oyentes, se hizo la traducción a LSC, la cual posteriormente se sometió a dos jurados que corroboraron que era clara, amplia y suficiente. El cuestionario finalmente fue aplicado por la guía intérprete del paciente con el acompañamiento de la psicóloga, buscando así el ajuste razonable para el paciente sordociego. Este test consiste en dos tipos de historias: las historias sociales, relacionadas con estados mentales (ToM), y las historias físicas, relacionadas con acontecimientos físicos (control). Para cada tipo de historias existen 8 
ejemplos. Las historias se administraron de forma consecutiva e intercaladas según su tipo (i. e.: social, física, social, física, etc.). Tras cada historia se hizo una pregunta señada.

Para las puntuaciones se contabilizó, en primer lugar, el tiempo de realización de la tarea, es decir, el tiempo comprendido entre la presentación de la historia y el momento en que el sujeto autorizó la realización de la pregunta en LSC.

En segundo lugar, las respuestas dadas se puntuaron según el siguiente criterio: 0 para las respuestas incorrecta, 1 para las respuestas correctas parciales o expresadas de forma implícita, y 2 para las respuestas correctas completas o expresadas de forma explícita. Así pues, las puntuaciones para cada tipo de historias (sociales y físicas) oscilan entre un mínimo de 0 puntos y un máximo de 16 . Para este instrumento el coeficiente Alfa de Cronbach es de un .64, y la fiabilidad estuvo por encima del .80 para personas oyentes (Gil et al. 2012).

\section{Diagnóstico}

Según la entrevista clínica y los instrumentos empleados, el paciente presenta un diagnóstico de asesoramiento sexual (DSM 5, 2014) o consulta sexual no especificada [Z709] (CIE10, 2016).

\section{Consideraciones éticas}

Se garantizó el principio de anonimato y la participación voluntaria. El paciente firmó el consentimiento informado, el cual no solo fue transcrito a braille, sino que además fue explicado con el apoyo del guía intérprete en su lengua natural (LSC), respetando su autonomía lingüística.

El proceso de investigación se acogió al decreto 2369 del 22 de septiembre de 1997 del Ministerio de Salud, el cual solicita la no discriminación e igualdad en atención a personas sordas, además del derecho a participar en la vida científica, entre otras esferas de vida. 
Desde la ley colombiana 1090 se cumplen los artículos 50 y 55. Con base en la resolución 8430, este proceso representa un riesgo mínimo para el paciente, dando cumplimiento a los artículos 4, 5, 6, 8, 9, 10, 12, 14 y 26. Cabe aclarar que el sujeto de investigación, a pesar de su situación de sordoceguera, no contaba con representante legal, siendo él, en consecuencia, su propio representante.

\section{Resultados}

Tras el análisis, se concluyó que la conducta sexual disfuncional pudo haberse manifestado debido a la existencia de vulnerabilidades en áreas psicológicas y biológicas. En la primera, se encontró poca interacción con su familia: exceptuando una persona, los demás integrantes no tenían dominio de Lsc, lo cual impide la comunicación óptima y limita la educación social, sexual y respecto a los demás temas relacionados al desarrollo. Estos aspectos, sumados a la ausencia de una asignatura continúa dedicada a temas sexuales, provocaron limitaciones de representaciones mentales asociadas a la sexualidad, de modo que el paciente contaba con información insuficiente para contemplar la situación y el significado social de su conducta sexual al momento del evento de tocamiento. Otro aspecto contribuyente fue haber sido víctima de tocamiento en la infancia; hecho que no tuvo una intervención psicoterapéutica inmediata.

En el aspecto de la vulnerabilidad biológica, se debe comprender que el paciente por haber nacido sordo tuvo dificultades para acceder a la información total del contexto y la adquisición de la discapacidad visual, a los cinco años, redujo aún más su interacción directa con el entorno. Además, carecía de ajustes razonables a nivel gubernamental, social y tecnológico, por lo que la información de medios de comunicación o conversaciones cotidianas no estuvo disponible para él, conllevando que sus representaciones mentales quedaran limitadas. 
Asesoramiento sexual en persona sordociega a partir del control de contingencias y técnicas mentalizadoras

Figura 1. Aspectos contribuyentes para el desarrollo del trastorno

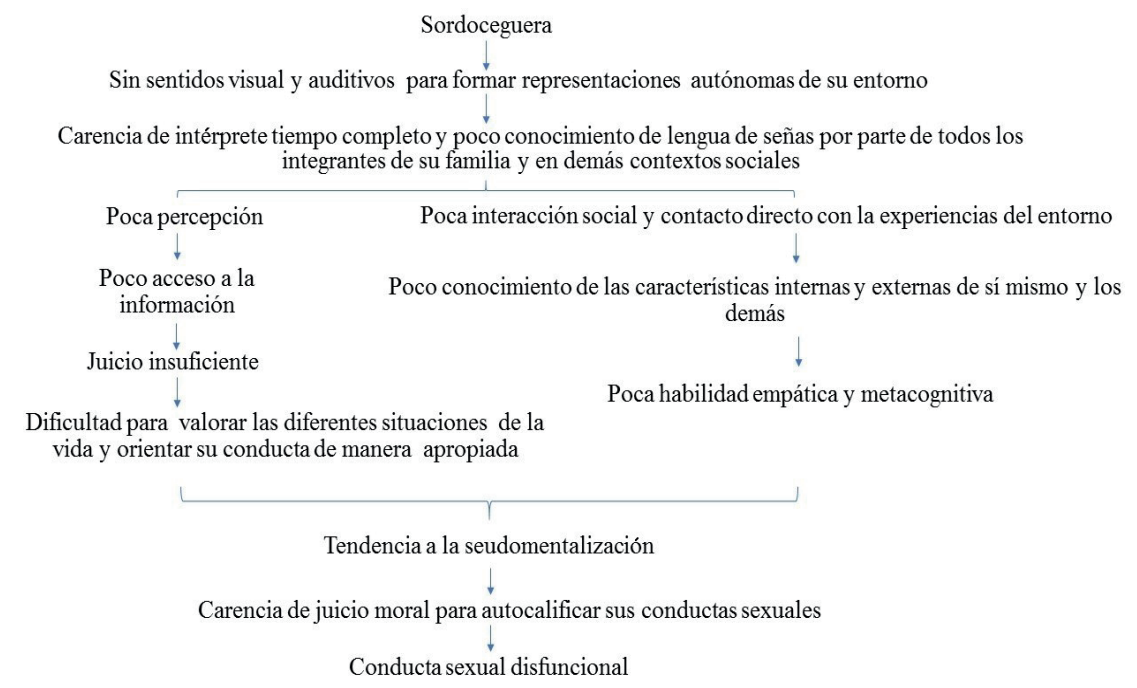

Como fortalezas se encontraron la culminación de sus estudios secundarios, su alto nivel en lengua de señas y su conocimiento básico del braille, lo que facilitó el proceso de psicoterapia. Además, uno de sus padres estuvo altamente comprometido y motivado con los procesos terapéuticos del paciente.

\section{Plan de tratamiento}

El caso se describió siguiendo el formato de presentación de casos del Instituto Beck. El modelo explicativo se realizó desde la evaluación de las áreas de mentalización propuestas por Bateman y Fonagy (2016), como se describió en la metodología, y la intervención fue orientada desde la terapia conductual, la educación sexual y la terapia basada en la mentalización. Para intervenir en la conducta sexual disfuncional (tabla 1) se realizó, en primer lugar, una entrevista tanto con los familiares como con el paciente, posteriormente se aplicó un programa de control de contingencias a la par que se ejecutaba un plan de educación sexual o psicoeducación, tanto para el paciente como para su hermano, en sesiones individuales. Por último, se finalizó con una terapia basada en la mentalización. 


\section{Tabla 1}

Plan de tratamiento

\begin{tabular}{|c|c|c|c|c|}
\hline Problema & Objetivo & Subobjetivo & Intervención & Semana nro. \\
\hline \multirow{7}{*}{$\begin{array}{l}\text { Conductas de } \\
\text { tocamientos } \\
\text { con su } \\
\text { hermano }\end{array}$} & \multirow{7}{*}{$\begin{array}{l}\text { Eliminar la } \\
\text { conducta } \\
\text { sexual } \\
\text { disfuncional }\end{array}$} & $\begin{array}{l}\text { Analizar el } \\
\text { funcionamiento } \\
\text { de la conducta de } \\
\text { tocamiento }\end{array}$ & Entrevista clínica & \multirow{7}{*}{$\begin{array}{l}\text { Semana } \\
1-5\end{array}$} \\
\hline & & $\begin{array}{l}\text { Intervenir las } \\
\text { vulnerabilidades } \\
\text { del contexto } \\
\text { que motivan la } \\
\text { conducta sexual } \\
\text { disfuncional del } \\
\text { paciente }\end{array}$ & $\begin{array}{l}\text { Análisis funcional de la conducta de } \\
\text { tocamiento }\end{array}$ & \\
\hline & & $\begin{array}{l}\text { Psicoeducar a } \\
\text { los padres sobre } \\
\text { el programa de } \\
\text { control de contin- } \\
\text { gencias }\end{array}$ & Manipulación del medio ambiente & \\
\hline & & $\begin{array}{l}\text { Entrenar a los } \\
\text { padres en el pro- } \\
\text { grama de control } \\
\text { de contingencias y } \\
\text { manipulación de } \\
\text { consecuencias }\end{array}$ & $\begin{array}{l}\text { - Entrenamiento a los padres del } \\
\text { paciente en el programa de control } \\
\text { de contingencias } \\
\text { - Aplicación del programa de con- } \\
\text { trol de contingencias con el apoyo } \\
\text { de registros } \\
\text { - Promover en el hermano menor } \\
\text { del paciente conductas de auto- } \\
\text { cuidado }\end{array}$ & \\
\hline & & $\begin{array}{l}\text { Educar al paciente } \\
\text { en la semiótica de } \\
\text { términos psico- } \\
\text { sexuales en LsC }\end{array}$ & $\begin{array}{l}\text { Entrenamiento de asertividad por } \\
\text { medio de LS }\end{array}$ & \\
\hline & & $\begin{array}{l}\text { Explicar las fun- } \\
\text { ciones del cuerpo }\end{array}$ & $\begin{array}{l}\text { Configuración de las señas sexuales } \\
\text { y explicación de los significados de } \\
\text { cada una de ellas }\end{array}$ & \\
\hline & & $\begin{array}{l}\text { Explicación de } \\
\text { las etapas del desa- } \\
\text { rrollo y cambios } \\
\text { hormonales }\end{array}$ & $\begin{array}{l}\text { - Explicación de las funciones del } \\
\text { cuerpo por medio de la LSC y apo- } \\
\text { yo lúdico en tercera dimensión } \\
\text { - Explicación de etapas del desarrollo } \\
\text { y cambios hormonales según el } \\
\text { género en LSC } \\
\text { - Explicación del desarrollo físico } \\
\text { - Explicación del control de esfín- } \\
\text { teres, control sexual y prácticas } \\
\text { masturbatorias }\end{array}$ & \\
\hline
\end{tabular}


- Entrenamiento en la inclusión de emociones y pensamientos dentro de su gramática de lengua de señas táctil.

- Entrenamiento en identificación de pensamientos propios y de los demás

\begin{tabular}{ll}
\hline $\begin{array}{l}\text { Aumentar la } \\
\text { representación de } \\
\text { las expresiones } \\
\text { gestuales y de }\end{array}$ & $\begin{array}{l}\text { - Exploración táctil de los cambios } \\
\text { faciales durante los procesos comu- } \\
\text { nicacionales }\end{array}$ \\
quienes le rodean & - Análisis de historias ToM \\
& - Entrenamiento en predicción de \\
& $\begin{array}{l}\text { los pensamientos y emociones de } \\
\text { los demás por medio de cuentos o } \\
\text { relatos infantiles }\end{array}$
\end{tabular}

Disminuir la - Juego de roles con exploración tendencia de con- facial táctil, promoviendo la siderar a los otros inferencia de las experiencias cogen términos de sí nitivas y emocionales de diferentes Semana mismo (aumento individuos ante la misma situación. $\quad 20-37$ de empatía) - Juego de roles que promuevan la identificación de lo que podría sentir el otro según sus reacciones

Nota. LS = lengua de señas; LSC = lengua de señas colombiana; ToM = teoría de la mente.

En relación con sus cambios sintomáticos: al inicio del tratamiento el paciente presentaba conductas sexuales disfuncionales únicamente durante el juego con su hermano menor, las cuales se extinguieron con la implementación del programa de control de contingencias y el plan de educación sexual (gráfico 1 y tabla 1). Durante la fase de evaluación era evidente el aplanamiento afectivo, el lenguaje gestual plano, el desconocimiento del real significado de algunas señas emocionales; únicamente eran reconocidas experiencias de emociones primarias. Con la aplicación de la terapia basada en la mentalización adaptada a la LS, y la comprensión de la cultura sordociega por parte del terapeuta, al trabajar en cada una de las áreas de mentalización el paciente incorporó una adecuada configuración con mayor expresividad facial de las señas relacionadas a las emociones, además de la identificación de la experimentación y el real significado de algunas emociones secundarias. 
Interpersonalmente no ocurrieron cambios significativos, debido a que durante el tratamiento solo uno de sus familiares dominaba la lengua de señas colombiana. Al finalizar el tratamiento, el estilo de juego adecuado con su hermano aumentó. De otro lado, cognitivamente el paciente empezó su tratamiento sin capacidad de juicio para evaluar sus conductas como inapropiadas, careciendo de representaciones mentales frente a temas socio-sexuales. Estas representaciones fueron consolidadas con el plan de educación sexual desde la semiótica de la usc (tabla 1).

Con relación a su capacidad mentalizadora, el paciente adquirió la habilidad de acompañar sus señas con lenguaje gestual y corporal e identificación de sus sensaciones corporales; aumentó, también, su interés por las características externas de los demás. Aunado a ello comprende y sabe identificar algunas emociones propias y comenzó a inferir los estados emocionales de los demás. Estos avances en la mentalización se logran identificar con los resultados obtenidos en la prueba de Historias extrañas de Happé. En la aplicación pretest El paciente obtuvo una puntuación de 1/16 en historia sociales y 3/16 en historias físicas; en el pos-test, una puntuación de 7/16 en historias sociales; no obstante, en las historias físicas continuó con una puntuación de 3/16.

Actualmente al paciente aún le cuesta integrar el conocimiento afectivo de sí mismo y de los demás con el conocimiento cognitivo. Continúa también con algunas dificultades para conectarse con el contexto social y el medio ambiente, además presenta dificultad para percibir las impresiones que él puede transmitirle a los demás desde sus comportamientos o actitudes. Por ello, se ha acordado con el paciente y sus familiares dar continuidad al tratamiento basado en la mentalización, dado que estas capacidades tienen una prospectiva de progreso con procesos adecuados de estimulación en sus cuatro dimensiones (conocimiento interno y externo de sí y de los otros).

Durante el proceso se presentaron algunos obstáculos como la poca accesibilidad del paciente a técnicas que implicasen el español escrito, su dificultad para el insight y la ausencia de contacto directo con su contexto. 


\section{Discusión}

Actualmente el paciente extinguió sus conductas de tocamiento, debido a la aplicación del programa de control de contingencias, cuya efectividad en población con discapacidad se presentó en la investigación de Athens y Vollmer (2010). En ella encontraron que este programa es útil para reducir el comportamiento problemático y aumentar el comportamiento apropiado en niños en situación de discapacidad cognitiva. Los programas de control de contingencias permiten manipular el entorno hasta que el paciente lo identifica como preventivo, predictivo y reactivo, de tal manera que logra modificar las consecuencias y genera una puerta de entrada, en el asesoramiento sexual, para que la persona viva su sexualidad de un modo regulado socialmente. Asimismo, el paciente logró la adquisición de representaciones mentales con relación a temas de educación sexual; estrategia también planteada por Sciacca (2017) para desarrollar programas educativos con niños y jóvenes sordociegos como medio de promoción de la salud sexual. Las personas sordociegas sin acceso a la información de un programa de educación sexual tendrán pobres habilidades de toma de decisiones en situaciones de expresión sexual, privacidad, cortejo y demás circunstancias asociadas a la socio-sexualidad. Aunque no es posible predecir el inicio de la vida amorosa del paciente, continuar la intervención tiene lugar, pues esta permitirá el desarrollo de más habilidades metacognitivas y una mayor conciencia interpersonal

Aunado a lo anterior, el paciente logró el desarrollo de algunas habilidades mentalizadoras gracias a la terapia basada en la mentalización. Este mismo método fue aplicado por Bateman y Fonagy (2016) en la Universidad de Oxford con el fin de lograr procesos de aumento del entendimiento de la propia mente y la de los demás, demostrando en sus investigaciones altos niveles de eficacia de esta terapia para mejorar el desempeño en las cuatro dimensiones mentalizadoras, relacionadas con el conocimiento interno y externo de sí mismo y de los demás.

Durante el estudio se presentaron algunas limitaciones. En el aspecto de la evaluación, en Colombia no se encuentran pruebas disponibles y validadas, con un constructo asociado con la teoría de la mente, para ser aplicadas en 
LSC. Además, las pruebas adaptadas al español que valoran habilidades relacionadas con la ToM no presentan una medición integral de todos los aspectos que componen la mentalización.

Es importante que para trabajos futuros la investigación se centre en la adaptación cultural de instrumentos psicométricos en LSC, no solo para evaluar ToM, también para apoyar el diagnóstico de trastornos psiquiátricos. Aunado a ello, el terapeuta que trabaja con este tipo de población debe conocer la cultura de la comunidad sorda, tener competencias para la habilitación de procesos cognitivos y habilidades metacognitivas en la población sordociega, en las cuales, además, se presentan particularidades dactilológicas, comunicacionales e intelectuales entre sujetos al interior de la misma comunidad. La familia de una persona sordociega podría ser un aliado del proceso psicoterapéutico si es conocedora de la LSC, pero si no son personas usuarias de esta lengua, su rol como aliado en la terapia tendrá que verse limitado a instructivos conductuales, o de cambios ambientales al interior del hogar, sugeridos por el terapeuta.

El programa de control de contingencias, acompañado de la ejecución del plan de educación sexual, desde la semiótica de la Lsc, y la aplicación de la terapia basada en la mentalización demostraron efectividad en la intervención en asesoramiento sexual, para, en primer lugar, anular la conducta sexual disfuncional, desarrollar representaciones socio-sexuales y aumentar la capacidad de juicio moral en relación con el comportamiento sexual. El paciente, con las aplicaciones de estas tres intervenciones, logró mayor comprensión de la realidad, autorregulación y capacidad de empatía, aunque esta última requiere de más sesiones desde la terapia de mentalización para alcanzar el máximo nivel de desarrollo según la psicoevolución del paciente.

Por último, cabe señalar que los resultados de este artículo no deben ser generalizados, debido a que la metodología de caso único no puede ser tomada como un referente psicoterapéutico de emisión de conceptos respecto a una población global. 


\section{Referencias}

American Psychological Association. 2014. Guía de consulta de los criterios diagnósticos del DSM-5 (5. ${ }^{\text {ta }}$ ed.). Washington: APA, 2014.

Anderson, T. y Cole, T. (2016). Sexual Counseling of the Physically Disabled. Postgraduate Medicine, 58(1), 117-123. doi: https://doi.org/10.1080/00325481.19 75.11714102

Athens, E. S. y Vollmer, T. R. (2010). An Investigation of Differential Reinforcement of Alternative Behavior Without Extinction. Journal of Applied Behavior Analysis, 43(4), 569-589. doi: https://doi.org/10.1901/jaba.2010.43-569

Bateman, A. y Fonagy, P. (2016). Mentalization-Based Treatment for Personality Disorders: A Practical Guide. New York: Oxford University Press.

Colom, F. (2011). Psicoeducación, el litio de las psicoterapias. Algunas consideraciones sobre su eficacia y su implementación en la práctica diaria. Revista Colombiana de Psiquiatría, 40, 147-165. doi: https://doi.org/10.1016/S00347450(14)60200-6

Domjan, M. (2010). Principios de aprendizaje y conducta (6. ${ }^{\text {ta }}$ ed.). México: Cengage Learning.

Gil, D., Fernández, M., Bengoechea, R., Arrieta, M. (2012). Teoría de la mente en esquizofrenia: su relación con síntomas positivos y negativos. Informaciones psiquiátricas, 210, 393-404. Recuperado de http://www.informacionespsiquiatricas.com/anteriores/210_inf\%20psiq.pdf

Kassa, T. A., Luck, T., Bekele, A., y Riedel-Heller, S. G. (2016). Sexual and Reproductive Health of Young People With Disability in Ethiopia: A Study on Knowledge, Attitude and Practice: A Cross-Sectional Study. Global Health, 12(5). doi: https://doi.org/10.1186/s12992-016-0142-3

Lahera, G. (2007). Teoría de la mente y desarrollo de sintomas psicóticos en el trastorno bipolar (Tesis doctoral). Universidad de Alcalá, España.

Lehane, C. M., Dammeyer, J., Hovaldt, H. B., y Peter E. (2017). Sexuality and Well-Being Among Couples Living With Acquired Deafblindness. Sex Disabil., 35, 135-146 doi: https://doi.org/10.1007

Ley 982. Por la cual se establecen normas tendientes a la equiparación de oportunidades para las personas sordas y sordociegas y se dictan otras disposiciones. Agosto de 2005 Recuperado de https://www.secretariajuridica.gov.co/transparencia/marco-legal/normatividad/ley-982-2005 
Asesoramiento sexual en persona sordociega a partir del control de contingencias y técnicas mentalizadoras

López, S. (2002). Sexo y Afecto en personas con discapacidad. España: Biblioteca Nueva.

Martínez-Taboas, A. (2014). Prácticas psicológicas basadas en la evidencia: beneficios y retos para Latinoamérica. Revista Costarricense de Psicología, 33(2), 63-78. Recuperado de http://www.rcps-cr.org/openjournal/index.php/RCPs/article/ view/33

Organización Mundial de la Salud (2016). Manual de codificación Clasificación internacional de enfermedades (CIE IO). Recuperado de https://eciemaps.mscbs.gob. es/ecieMaps/browser/index_10_mc.html

Perpiñá, C. (2012). Manual de entrevista psicológica. Saber escuchar, saber preguntar (coord.). Madrid: Ediciones Pirámide.

Quintana, I. (2004). Deficiencia auditiva y teoria de la mente un estudio de las variables que influyen en la comprensión de falsa creencia en niños y adolescentes sordos (Tesis doctoral). Universidad de Málaga, España. Recuperado de http://www.biblioteca.uma.es/bbldoc/tesisuma/16764262.pdf.

Sciacca, R. (2017). Transición hacia la vida adulta de jóvenes con sordoceguera: herramientas para garantizarla. Educ@ción en Contexto, 3, 140-166. Recuperado de https://dialnet.unirioja.es/servlet/articulo? codigo $=6296630$

Wellman, H. M. y Peterson, C. C. (2013). Theory of Mind, Development, and Deafness. En S. Baron-Cohen, M. Lombardo, y H. Tager-Flusberg (Eds.), Understanding Other Minds: Perspectives from Developmental Social Neuroscience (pp. 51-71). Oxford: Oxford University Press. 